\section{p63 expression in sarcomatoid/metaplastic carcinomas of the breast}

Sir: In the paper by Kurian and Al-Nafussi, ${ }^{1}$ describing a series of sarcomatoid/metaplastic carcinoma of the breast, the authors stress the difficulties in differentiating a bona fide case of monophasic sarcomatoid tumour of the breast from its mimics, such as phyllodes tumours or breast sarcomas. ${ }^{1}$ Briefly, the role of immunohistochemical markers, such as low molecular weight cytokeratins and epithelial membrane antigen, in the recognition of these rather unusual and tricky neoplasms was emphasized. ${ }^{1}$ We would like to add some promising data regarding p63, a novel basal/myoepithelial cell immunohistochemical marker that may help in this differential diagnosis and may also give new insights on the histogenesis and differentiation of sarcomatoid/metaplastic carcinomas of the breast.

We have been analysing p63 (clone 4A4, dilution 1:150; Neomarkers, Freemont, CA, USA) expression in several types of breast neoplasms. ${ }^{2}$ p63 is a p53 homologue whose gene is located on $3 \mathrm{q} 27^{2,3}$ At variance with $p 53$ gene, which encodes an unique 53-kDa protein, $p 63$ gene encodes six isoforms which differ in the $C$-terminal $(\alpha, \beta$, and $\gamma)$ and in the $\mathrm{N}$-terminal (transactivating and $\Delta \mathrm{N}$-isoforms). ${ }^{2,3}$ While TA-isoforms are able to promote the transcription of p53-reporting genes, $\Delta \mathrm{N}$-isoforms are unable to do so. $^{2-4}$ Interestingly, $\Delta \mathrm{N}$-isoforms are consistently expressed in the nuclei of myoepithelial/basal cells of the breast, as well as in basal cells of several multilayered epithelia ${ }^{2-4}$ which may be an alternative mechanism to overcome p53-driven cell cycle arrest and apoptosis. ${ }^{2-4}$

$\mathrm{We}^{2}$ and others ${ }^{4-6}$ have observed a strong and diffuse p63 expression in the nuclei of normal myoepithelial cells of breast lobules and ducts, as well as in the cells of myoepithelial tumours, such as sclerosing papilloma, adenomyoepithelioma, adenoid-cystic carcinoma, epithelial-myoepithelial carcinoma and malignant adenomyoepithelioma. ${ }^{2,4}$ Interestingly, $\mathrm{we}^{2}$ and others $^{4,5}$ also observed p63 expression in cases of monophasic sarcomatoid/metaplastic carcinomas of the breast. An eye-catching feature was a rather strong and consistent p63 expression in the nuclei of spindle and epithelioid neoplastic cells in bona fide cases of mono- and biphasic sarcomatoid/metaplastic carcinomas of the breast (Figure 1). The majority of these cells also co-expressed cytokeratins; however, some spindle cells showed p63 expression only. p63 seems to be a good myoepithelial marker, because stromal cells, fibroblasts, and myofibroblasts observed in normal
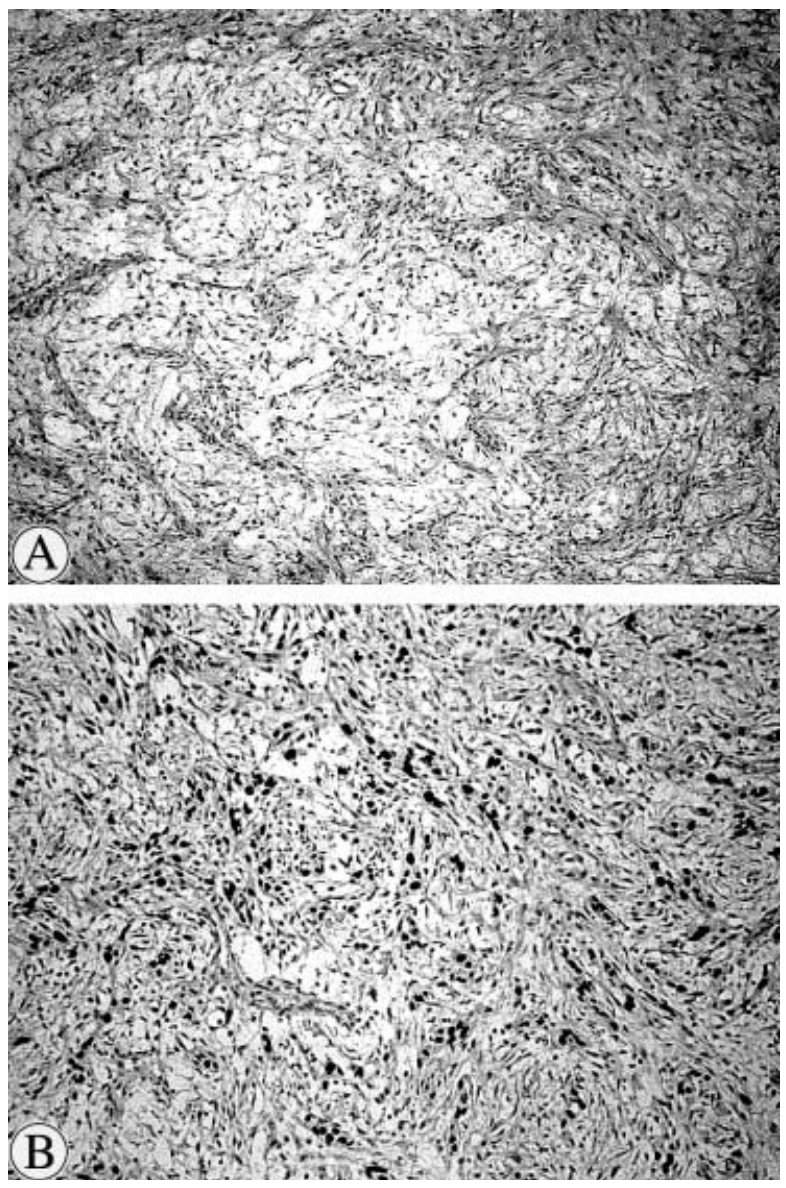

Figure 1. Medium power magnification of a monophasic and blandlooking sarcomatoid/metaplastic carcinoma of the breast. A, Note the presence of myxoid areas and the deceiving bland appearance of the cells (H\&E). B, p63 immunostaining highlighting the nuclei of neoplastic cells arranged in strands and cords. (Streptavidinbiotin-peroxidase/DAB.)

breast, fibromatoses, fibroadenomas and phyllodes tumours fail to express p63, as observed by our group (Reis Filho et al., manuscript submitted) and Barbareschi et al. ${ }^{4}$

p63 expression in normal breast tissue and neoplasms of the breast is not a fully characterized phenomenon. Based on double-immunostaining studies, the expression of p63 and smooth-muscle markers, such as $\alpha$-smooth muscle actin, calponin, and smooth muscle myosin heavy chain, Barbareschi et al. ${ }^{4}$ demonstrated that a subset of p63+ cells co-express smooth-muscle markers, while others do not. The p63+ smooth-muscle marker-positive phenotype probably represents the usual immunophenotype of myoepithelial cells, while p63+ smooth-muscle marker-negative is probably what could be expected to be expressed by basal cells. ${ }^{2,4}$ The presence of p63 immunoreactivity seems to point 
towards a putative myoepithelial/basal cell histogenesis/ differentiation in some types of sarcomatoid/metaplastic carcinomas of the breast. ${ }^{2,4,5}$

Based on our preliminary results ${ }^{2}$ and on Barbareschi et al. ${ }^{4}$ and Wang et al. ${ }^{5}$ findings, we would like to stress that p63 might be included in immunohistochemical panels designed to diagnose peculiar breast neoplasms that putatively show a myoepithelial histogenesis. p63 may also play a major role in the diagnosis of sarcomatoid/metaplastic carcinomas of the breast, because it is consistently expressed in these tumours. ${ }^{2}$ Moreover, these findings warrant that the role of p63 pathway should be investigated in neoplasms showing a basal/myoepithelial cell immunophenotype, because it seems that tumours with a basal cell phenotype are associated with an aggressive behaviour $^{7}$ and that $\Delta \mathrm{N}-\mathrm{p} 63$ over-expression might be an alternative mechanism to overcome p53 tumour suppressor functions.

\author{
J S Reis-Filho \\ F C Schmitt ${ }^{1}$ \\ School of Health Sciences, \\ University of Minho, \\ Braga and Institute of Molecular Pathology \\ and Immunology, \\ University of Porto (IPATIMUP), Porto, and \\ ${ }^{1}$ IPATIMUP and Medical Faculty, \\ University of Porto, Porto, \\ Portugal
}

1. Kurian KM, Al-Nafussi A. Sarcomatoid/metaplastic carcinoma of the breast: a clinicopathological study of 12 cases. Histopathology 2002; 40; 58-64.

2. Reis-Filho JS, Schmitt FC. Taking advantage of basic research: p63 is a reliable myoepithelial and stem cell marker. Adv. Anat. Pathol. 2002; 9; 280-289.

3. Yang A, McKeon F. p63 and p73: p53 mimics, menaces and more. Nat. Rev. Molec. Cell Biol. 2000; 1; 199-207.

4. Barbareschi M, Pecciarini L, Cangi MG et al. p63, a p53 homologue, is a selective nuclear marker of myoepithelial cells of the human breast. Am. J. Surg. Pathol. 2001; 25; 10541060 .

5. Wang X, Mori I, Tang W et al. Metaplastic carcinoma of the breast: p53 analysis identified the same point mutation in the three histologic components. Mod. Pathol. 2001; 14; 1183-1186.

6. DiRenzo J, Signoretti S, Nakamura N et al. Growth factor requirements and basal phenotype of an immortalized mammary epithelial cell line. Cancer Res. 2002; 62; 89-98.

7. Sorlie T, Perou CM, Tibshirani R et al. Gene expression patterns of breast carcinomas distinguish tumor subclasses with clinical implications. Proc. Natl Acad. Sci. USA 2001; 98; 10869-10874. 